Research Journal of Applied Sciences 13 (9): 552-558, 2018

ISSN: $1815-932 \mathrm{X}$

(C) Medwell Journals, 2018

\title{
Artificial Intelligence Algorithm for Autonomous Movement of a Smart Wheelchair
}

\author{
Mauricio Plaza Torres and Fredy David Bernal Castillo \\ Faculty of Engineering, Universidad Militar Nueva Granada, Carrera 11\#101-80, Bogota, Colombia
}

\begin{abstract}
Nowadays there are many engineering applications in the medical field, including pathology diagnostic systems, magnetic resonance imaging, physiological signal monitoring system, upper and lower limb prostheses and orthoses, all these developments aim to improve the quality of life of the human beings. The present document shows the development of an artificial intelligence genetic algorithm through which the trajectories of a smart wheelchair in a known environment are planned, to transfer a patient with upper and lower limb mobility problems or with spinal cord injury. The device assists him in his movement from a starting point to a goal point to which the user wants to arrive. The trajectory generator algorithm was developed in MATLAB Software, in addition to finding the shortest possible route between the two points, implements a safety function that influence in final decision of the trajectory to be performed in order to prevent collisions with obstacles that can be avoided by the wheelchair but possibly have contact with parts of the patient's body that are outside it. With the generation of the recursion algorithm, the processing times for obtaining trajectories were improved for the recursion algorithm the average time taken to find a point of a feasible trajectory is $0.085 \mathrm{sec}$ while for the algorithm without recursion is $0.168 \mathrm{sec}$.
\end{abstract}

Key words: Artificial intelligence, algorithms, wheelchairs, spinal cord injuries, software, genetic variety

\section{INTRODUCTION}

According to the World Health Organization and the World Bank, it is estimated that the world population with some type of disability is equivalent to $15 \%$ of the total population, that is more than a billion people (Agah, 2013), specifically the number of people that require a wheelchair are approximately $131,800,000$ in total in Latin America are very few users with these pathologies have a wheelchair movement system and very few cities have mobility systems and take into account the needs of people with lower-train disabilities (Caceres et al., 2017).

Artificial intelligence has contributed to solve many problems in today's world is in multiple industries and applications ranging from voice assistants on our mobile phones to industrial robotics in the manufacture of products and high-risk jobs, including applications such as disease detection, customer service, transportation systems, games, economic decision making, among others.

Specifically, in medicine there is a wide variety of applications that use artificial intelligence algorithms to solve problems and make decisions in search of human well-being, this is the case with expert systems for the diagnosis of diseases, assisted surgery robots, automated radiologist, lifestyle monitoring and management systems, nutrition, etc. This research aims to generate an artificial intelligence algorithm to emulate the optimal movement of a smart wheelchair with a starting point and a goal which learns from its environment and can be used by people with limited lower body mobility (De Garcia, 2006).

The development of the algorithm will take place through the artificial intelligence programming technique called Genetic algorithms which are adaptive methods based on Darwin's natural selection theory (which states the survival of the strongest) applied to the search and solution of problems in our specific case, the emulation of the search for optimal trajectories for the movement of a smart wheelchair in environments with obstacle (Goldberg, 1989).

The purpose of the research is to implement emerging technologies to help people with disabilities in lower limbs by means of the development of an algorithm that allows the emulation of smart wheelchair movement, so that, it can be implemented in future physical tests with patients who use conventional wheelchairs and so that, they can move autonomously in an obstructed environment without third-party assistance. The search for trajectories was carried out using artificial intelligence algorithms in search of the well-being of people in a wheelchair condition.

The scope of the proposal, refers to emulating a mapping system with trajectories and solving the possible paths of the wheelchair by means of the technique of genetic algorithms, so that, it can move in this way without colliding with obstacles.

Corresponding Author: Mauricio Plaza Torres, Faculty of Engineering, Universidad Militar Nueva Granada, Carrera 11\#101-80, Bogota, Colombia 


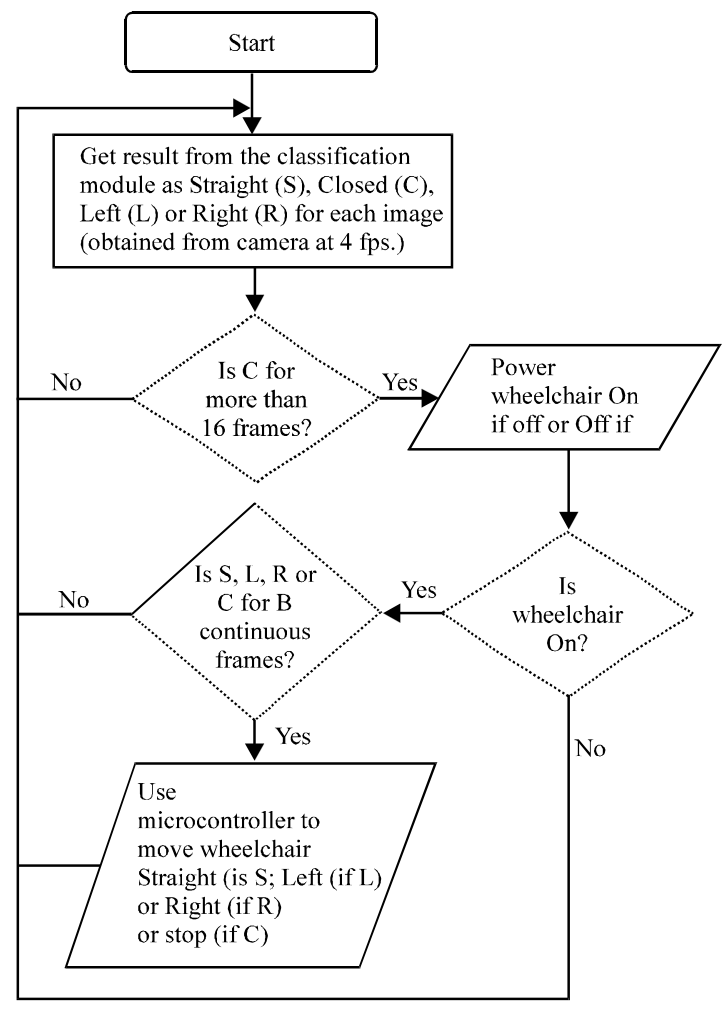

Fig. 1: Algorithm for wheelchair control

The state of the art includes artificial intelligence applications for the control of wheelchairs, as in 2006 the University of Brussels in Belgium (Jiao et al., 2018) developed a method based on grouping Genetic algorithms for the transport of disabled people in hospitals, shopping malls and different enclosed spaces. Using this technique, a stochastic search based on random operators is performed to combine different solutions and produce the most optimal solutions.

Rajesh and Mantur (2017) of the Karnata Institute of Technology in Bangladesh developed a wheelchair that uses the activity of the brain (electroencephalography signals) and the eye (electrooculography signals), so that, these signals through the artificial intelligence technique called Deep Learning allow the movement of patients with quadriplegia, the algorithm used for the wheelchair system works through a camera that captures the patient's eye activity at four frames per second, if the user looks at the same point for 8 frames in a row, i.e., $2 \mathrm{sec}$, he will move in that direction, if, however, he closes his eyes and the system remains in a static position, the programming logic of the algorithm for controlling the wheelchair is shown in Fig. 1.

In 2018, Rekiek et al. (2006) in China, Jiao's research group performed an adaptive polymorphic algorithm using

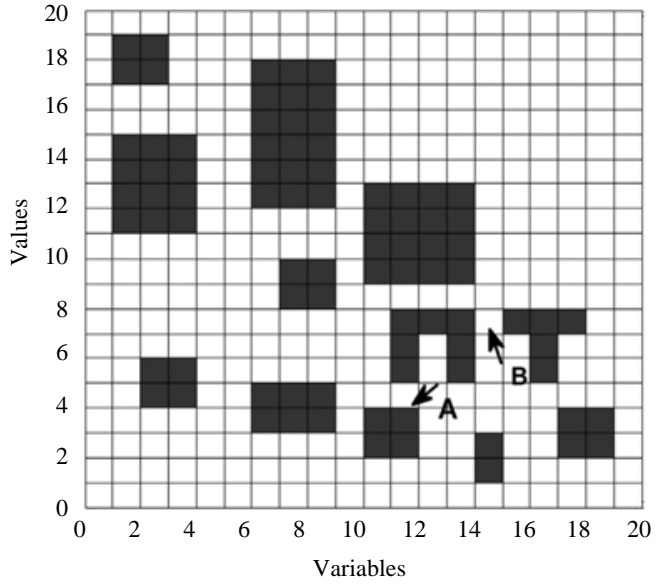

Fig. 2: Genetic algorithm applied to obtain the most optimal trajectory

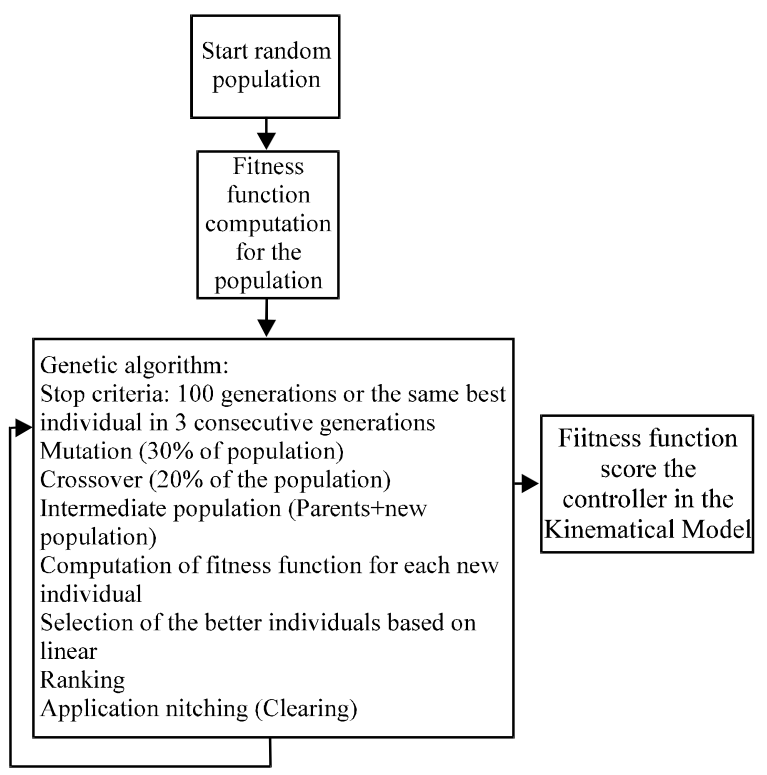

Fig. 3: Structure of the Genetic algorithm implemented using NEAT principles

an ant swarm intelligence method which plans the trajectories of a smart wheelchair, so that, people in wheelchair condition can benefit from the automated navigation system, Fig. 2 shows the emulation of the algorithm for the solution in the search for the most optimal trajectory avoiding obstacles and closed roads.

In 2017 (WHO., 2011) a hybrid reactive navigation controller was developed for a non-holonomic robot applicable for wheelchair autonomy to improve the quality of life of people with disabilities, the controller was designed using the "Neuro Evolution of Augmented Topologies" (NEATS) algorithm as shown in Fig. 3. 


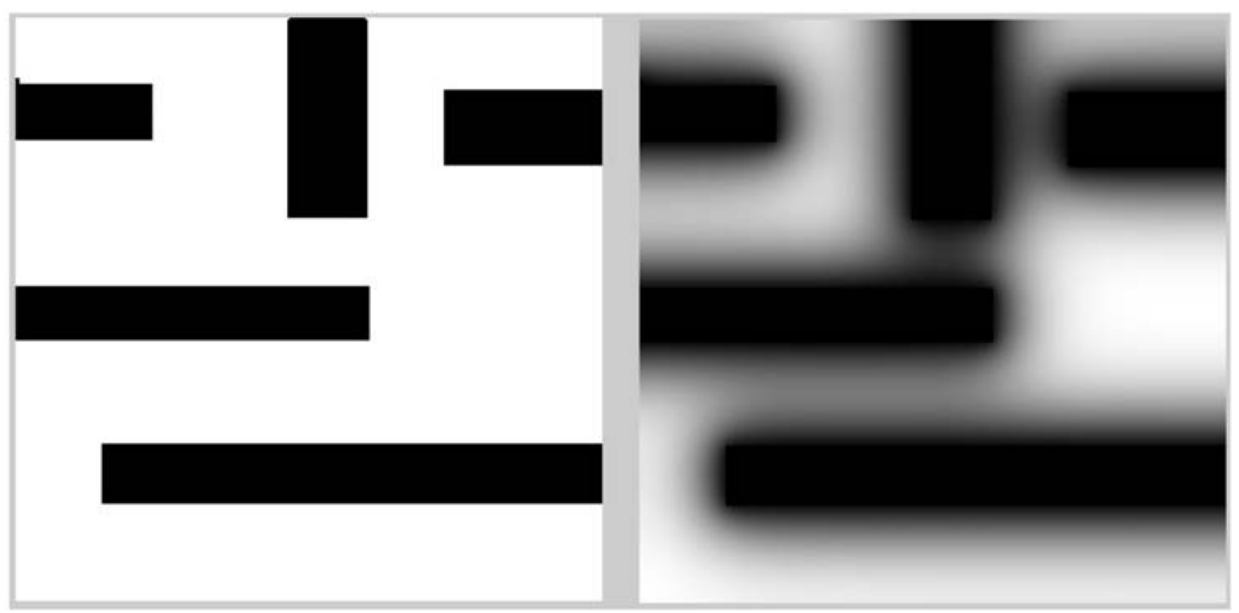

Fig. 4: Original map (left) and map with influence of obstacles (right)

\section{MATERIALS AND METHODS}

Different artificial intelligence techniques were identified from assessments of computational consumption, learning time and finally execution time, which is why it was decided to use the Genetic algorithms technique to develop with recursion, since, as described in the state of the art there are several developments of artificial intelligence for the automation of smart wheelchairs but they do not make use of genetic algorithms with recursive programming during their development.

At the heart of the research described in this document is the trajectory planner, the problem is simple, a wheelchair must be able to move in a known environment, moving a patient from position $\mathrm{A}$ to a position $\mathrm{B}$ in an environment where there are obstacles preventing a straight line trajectory.

The development of the trajectory generation algorithm differs from those found in the state of the art, for comparatively similar problems, on the relative importance of the safety function of the trajectory, defined as the measure of the distance from the trajectory to the obstacles in these cases, it is imperative that the security function has a marked impact on the decisions that affect the generation of the trajectory, since, it must be ensured that patients are far away to avoid possible collisions with obstacles that can be avoided by the wheelchair but possibly have contact with parts of the patient's body that are outside it. We also want the trajectory to be as short as possible in order to reduce energy consumption due to movement and obviously, to reduce the time it takes.

It can be seen that the problem is a two-dimensional optimization problem in which we seek to optimize two functions that in fact, they adversely affect each other, on the one hand, greater safety means longer trajectories and short trajectories are also often unsafe, the problem was attacked by the influence of a circular filter to increase the influence of the obstacles on the possible routes of the map, so that, it is no longer binary (Fig. 4) shows the original map (left) and the obstacle map (right).

For the development of the trajectory planning algorithm, MATLAB and a $\mathrm{PC}$ with a 32 bit processor (Windows 8.1) were used as the programming environment.

\section{RESULTS AND DISCUSSION}

Figure 5 shows the data flow chart that explains the operation of the final algorithm developed, the "Load Map" function, performs a space recognition sweep in which the displacement trajectories will be searched, once the map is filtered by levels in grayscale, the data is discarded.

The heart of the program is the recursive function "Find-trajectory", the function creates something we'll call "Feasible space", The feasible space in this context is a set of randomly generated points in the search space that are not part of a prohibited area that is they are points on the map whose corresponding pixel value is 255 these points are distributed across the entire search space.

The find-trajectory function receives a cloud of points belonging to the feasible space search, a starting point of the comparison, the trajectory destination, the evaluation map and a search threshold (intended for non-binary maps but in grayscale, this parameter will define whether a section is considered an obstacle or not 
Res. J. Applied Sci., 13 (9): 552-558, 2018

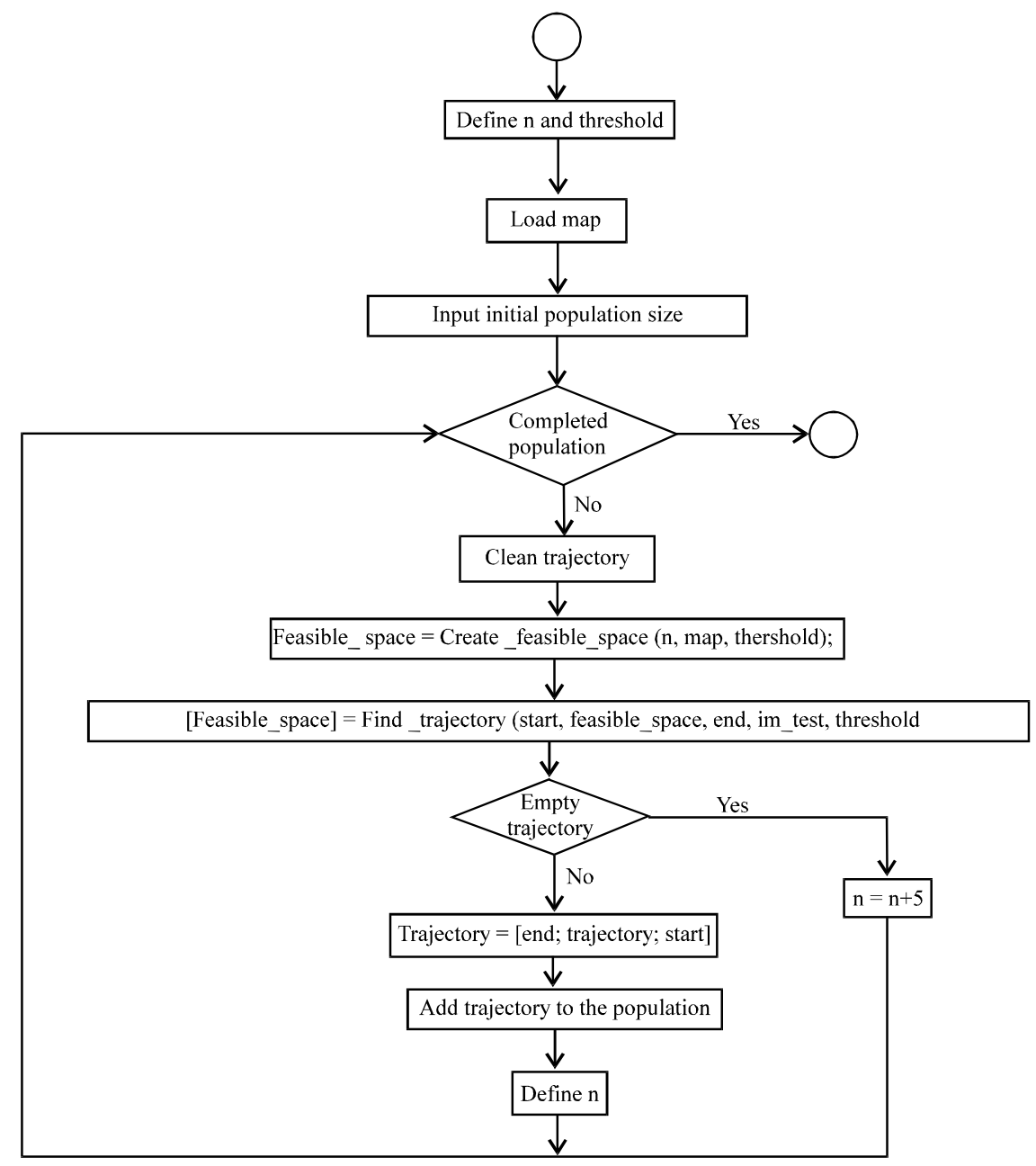

Fig. 5: Flowchart of the obtained algorithm

this parameter will define if a section is considered an obstacle or not), the algorithm starts by comparing the received point with the first point of the feasible space. The flowchart of the find-trajectory function can be seen in Fig. 6.

If there is a line of sight between the point received and the first point of feasible space, the algorithm checks whether that point in feasible space has line of sight at the end of the trajectory, if so, the algorithm ends, otherwise, that point becomes the new starting point, feasible space suppresses the current point creating a new feasible space that no longer has this point and the function calls itself, to repeat the process as shown in Fig. 7.

This kind of binary map, as shown in Fig. 7 are common in mobile robot trajectory planning problems, they are often the basis of collision planning and prevention algorithms and in our case is the basis of the processing in the navigation algorithm, this image is processed, so that, after ensuring that the obstacles have a minimum safety margin that prevents collisions with the wheelchair an additional factor with which we will evaluate our safety function will be included, the map will be degraded in grayscale, ensuring that the areas close to obstacles are close to black and areas far from obstacles remain in light tones.

In Fig. 8, we can see the emulated mapping of a route with several possible exits, the black rectangles represent the obstacles on the trajectory, including a safety area for the wheelchair to pass through (black) and an obstacle-free area (white) with the artificial intelligence algorithm implemented can be seen that the system analyzed the possible routes (red lines) and finally reached a solution.

Finally, Fig. 9 shows the average generation times used to generate a point of a feasible trajectory without recursion with recursion and with recursion and parallelism. 


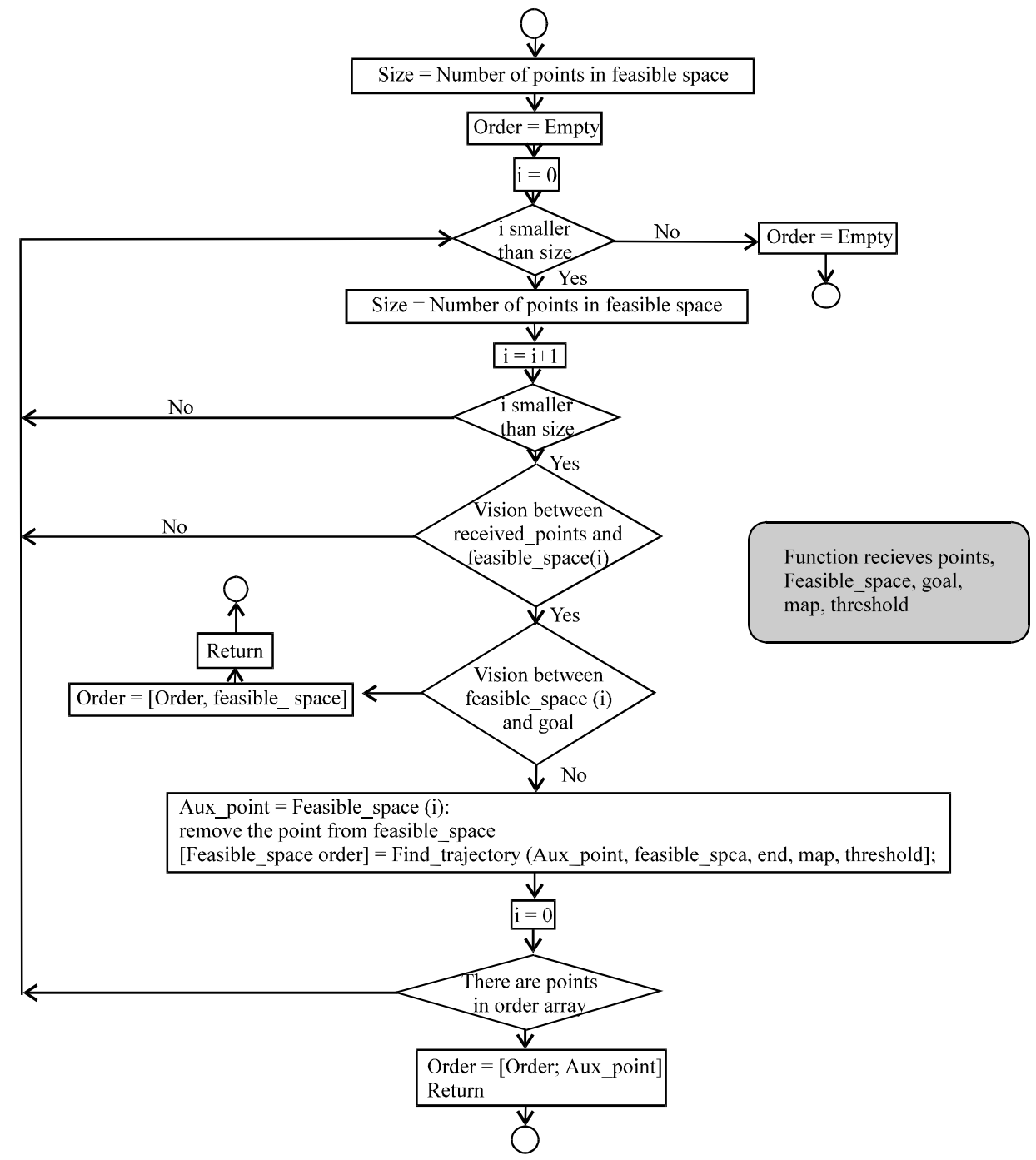

Fig. 6: Flowchart of the recursive "Find-trajectory" function

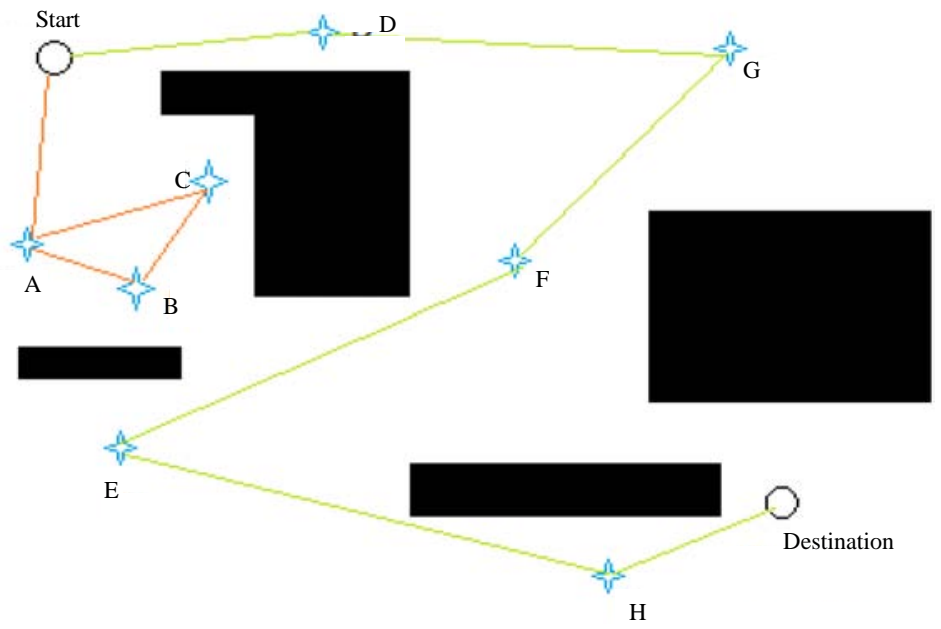

Fig. 7: Operation of the "Find-trajectory" function 


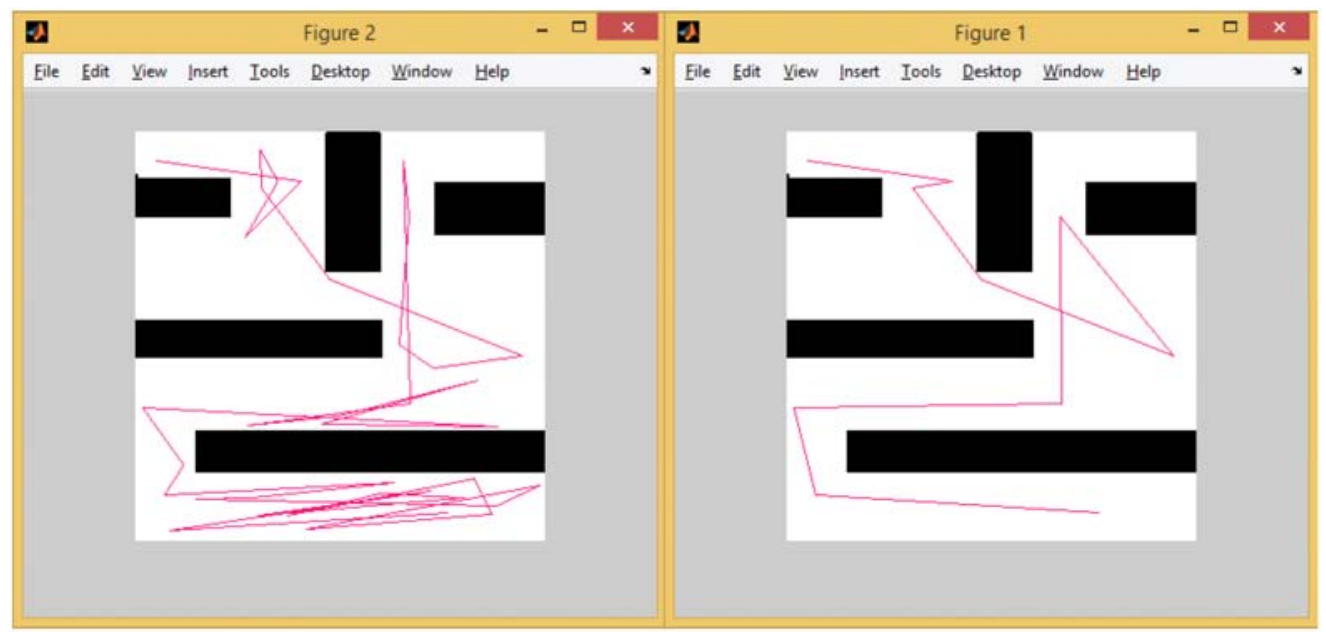

Fig. 8: Execution of the Genetic algorithm with recursion to a simulated mapping of regular house inside

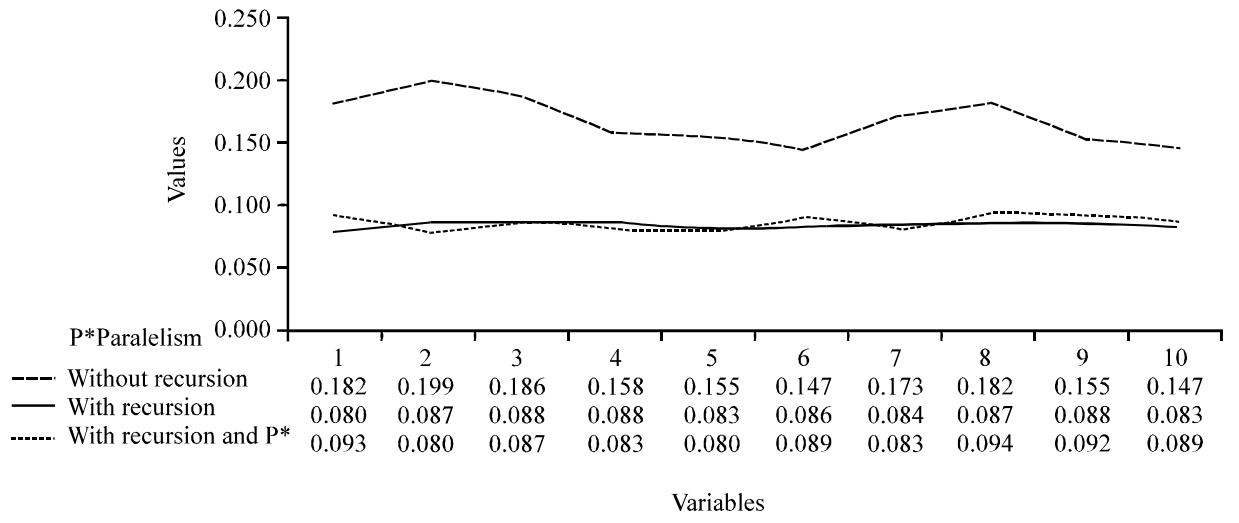

Fig. 9: Average time to generate one single point of a feasible trajectory

With the generation of the recursion algorithm, the processing times for obtaining trajectories were improved for the recursion algorithm the average time taken to find a point of a feasible trajectory is $0.085 \mathrm{sec}$ while for the algorithm without recursion is $0.168 \mathrm{sec}$.

The results suggest that implementing the artificial intelligence technique called genetic algorithms is a possible solution to problems in the search for optimal trajectories applied to autonomous movement systems and mobility applications for people with disabilities.

Recursion in terms of programming improves algorithms processing times and therefore, response speed of the autonomous mobility system in different environments with multiple obstacles. With the implementation of recursion within the program solved one of the biggest difficulties in the solution of searching for trajectories with artificial intelligence was solved, the enormous wear and tear on processing and time in maps or environments with very few obstacles.

\section{CONCLUSION}

The main innovation of the algorithm is the use of recursion programming, solving one of principal challenges of the project in searching trajectories: the limitation of genetic variety in scenarios with few obstacles.

\section{ACKNOWLEDGEMENTS}

Funding by IMP ING 2659 Universidad Militar Nueva Granada Research Unit.

\section{REFERENCES}

Agah, A., 2013. Medical Applications of Artificial Intelligence. CRC Press, Boca Raton, Florida, USA., ISBN:978-1-4398-8433-1, Pages: 492. 
Caceres, C., J.M. Rosario and D. Amaya, 2017. Approach of kinematic control for a nonholonomic wheeled robot using artificial neural networks and genetic algorithms. Proceedings of the 2017 International Conference and Workshop on Bioinspired Intelligence (IWOBI), July 10-12, 2017, IEEE, Funchal, Portugal, ISBN:978-1-5386-0851-7, pp: $1-6$.

De Garcia, P.S., 2006. [Approach to the Reality of People with Disabilities in Latin America]. CERMI, Brussels, Belgium, ISBN:9788461115020, Pages: 842 (In Spanish).

Goldberg, D.E., 1989. Genetic Algorithms in Search, Optimization and Machine Learning. 1st Edn., Addison-Wesley, Boston, Massachusetts, USA., ISBN:9780201015768, Pages: 412.
Jiao, Z., K. Ma, Y. Rong, P. Wang and H. Zhang et al., 2018. A path planning method using adaptive polymorphic Ant Colony algorithm for smart wheelchairs. J. Comput. Sci., 25: 50-57.

Rajesh, A. and M. Mantur, 2017. Eyeball gesture controlled automatic wheelchair using deep learning. Proceedings of the 2017 IEEE Region 10 Conference on Humanitarian Technology (R10-HTC), December 21-23, 2017, IEEE, Dhaka, Bangladesh, ISBN:978-1-5386-2176-9, pp: 387-391.

Rekiek, B., A. Delchambre and H.A. Saleh, 2006. Handicapped person transportation: An application of the grouping genetic algorithm. Eng. Appl. Artif. Intell., 19: 511-520.

WHO., 2011. The World Report on Disability. World Health Organization, Geneva, Switzerland, ISBN: 9789241548182, Pages: 325. 